\title{
Plasma B-Type Natriuretic Peptide Levels May Increase Because of Fat Mass Loss by Metformin or Sodium-Glucose Transporter 2 Inhibitors Treatment
}

\author{
Hidetaka Hamasakia, b, Hidekatsu Yanai ${ }^{\mathrm{a}}$
}

\begin{abstract}
Background: Cardiovascular disease (CVD) is the leading cause of death in type 2 diabetes. Metformin reduces cardiovascular events in obese patients with type 2 diabetes, and sodium-glucose transporter 2 (SGLT2) inhibitors decrease cardiovascular events in type 2 diabetic patients with established CVD. However, the underlying mechanisms behind the cardioprotective effects of metformin and SGLT2 inhibitors are unknown.
\end{abstract}

Methods: Fifteen patients with newly diagnosed type 2 diabetes receiving metformin monotherapy, and seven patients with type 2 diabetes receiving SGLT2 inhibitors combined with other hypoglycemic agents were studied. We investigated changes in glycemic control, plasma B-type natriuretic peptide (BNP) levels, and body composition, 3 and 6 months after starting metformin administration, and 3 months after starting SGLT2 inhibitor administration.

Results: Plasma BNP levels significantly increased after 3 months in both metformin and SGLT2 inhibitors treatment groups $(7.9 \pm 7.9 \mathrm{pg} /$ $\mathrm{mL}$ to $17 \pm 16.9 \mathrm{pg} / \mathrm{mL}, \mathrm{P}=0.012 ; 8.8 \pm 7.2 \mathrm{pg} / \mathrm{mL}$ to $15.5 \pm 14.3 \mathrm{pg} /$ $\mathrm{mL}, \mathrm{P}=0.018$, respectively). Fat mass significantly decreased in the first 3 months of metformin administration $(25.7 \pm 10.3 \mathrm{~kg}$ to $23.0 \pm$ $11.4 \mathrm{~kg}, \mathrm{P}=0.046$ ), while fat mass and visceral fat area decreased in three patients receiving SGLT2 inhibitors.

Conclusions: Plasma BNP levels increased because of fat mass loss caused by treatment with metformin and SGLT2 inhibitors. Our results suggest that metformin and SGLT2 inhibitors could reduce the risk of CVD by exerting cardioprotective effects through elevated BNP levels in patients with type 2 diabetes.

Keywords: Type 2 diabetes; Metformin; Sodium-glucose transporter 2 inhibitors; B-type natriuretic peptide; Body composition

Manuscript accepted for publication February 26, 2016

a Department of Internal Medicine, National Center for Global Health and Medicine Kohnodai Hospital, 1-7-1 Kohnodai, Ichikawa, Chiba, Japan ${ }^{b}$ Corresponding Author: Hidetaka Hamasaki, Department of Internal Medicine, National Center for Global Health and Medicine, Kohnodai Hospital, 1-7-1 Kohnodai, Chiba 272-8516, Japan. Email: hhamasaki78@gmail.com

doi: http://dx.doi.org/10.14740/jem333w

\section{Introduction}

Cardiovascular disease (CVD) is the major cause of death in type 2 diabetes. Having diabetes increases the risk of developing CVD and cardiovascular death (hazard ratio: 2.32) [1]. Previous studies suggest an approximate $15 \%$ reduction in the relative risk of CVD per $1 \%$ decrement in glycated hemoglobin A1c (HbA1c) [2]. To prevent CVD in the diabetic population, achieving optimal glycemic control is crucial.

Metformin is used in the initial treatment of type 2 diabetes. Metformin monotherapy reduced cardiovascular events in obese patients with type 2 diabetes compared to patients treated with lifestyle modifications [3]. Accumulating evidence suggests that metformin also has cardioprotective effects [3-5]. However, the specific mechanisms behind the cardioprotective effects of metformin are not clear.

Sodium-glucose transporter 2 (SGLT2) inhibitors are the novel oral glucose-lowering drugs, which inhibit renal proximal tubular glucose reabsorption. Recent clinical studies have shown that SGLT2 inhibitors improve glycemic control and obesity $[6,7]$. Furthermore, Zinman et al demonstrated that empagliflozin, an SGLT2 inhibitor, decreases cardiovascular events in type 2 diabetic patients with established CVD [8]. Cardiovascular events rapidly decreased in patients treated with empagliflozin, suggesting that an SGLT2 inhibitor confers some cardioprotective effects in addition to its beneficial effects on glycemic control, blood pressure, and body weight. However, as the authors stated, the mechanisms behind the cardiovascular benefits of empagliflozin are multidimensional, and the specific mechanisms behind the cardioprotective effects of SGLT2 inhibitors are unknown.

B-type natriuretic peptide (BNP) levels increase with the severity of left ventricular dysfunction and/or hypertrophy, which is useful for the screening of type 2 diabetic patients with cardiovascular complications [9], and in the diagnosis and prognosis of heart failure [10]. In the present study, we aimed to investigate the change in plasma BNP levels following the administration of metformin or SGLT2 inhibitors.

\section{Materials and Methods}

\section{Metformin treatment}

We studied 15 outpatients newly diagnosed with type 2 diabe- 
Table 1. Anthropometric and Biochemical Parameters at Baseline, 3 and 6 Months After Metformin Administration

\begin{tabular}{|c|c|c|c|c|c|c|}
\hline & Baseline & $\begin{array}{l}\text { After } 3 \\
\text { months }\end{array}$ & $\begin{array}{l}\text { After } 6 \\
\text { months }\end{array}$ & $\begin{array}{l}\text { P value (baseline } \\
\text { vs. after } 3 \text { months) }\end{array}$ & $\begin{array}{l}\text { P value (after } 3 \text { months } \\
\text { vs. after } 6 \text { months) }\end{array}$ & $\begin{array}{l}\text { P value (baseline } \\
\text { vs. after } 6 \text { months) }\end{array}$ \\
\hline $\mathrm{n}$ (men/women) & $15(7 / 8)$ & - & - & - & - & - \\
\hline Age (years) & $54.9 \pm 14$ & - & - & - & - & - \\
\hline Weight (kg) & $74.5 \pm 15.6$ & $68.6 \pm 13.1$ & $70.6 \pm 14.6$ & 0.068 & 0.114 & 0.043 \\
\hline BMI $\left(\mathrm{kg} / \mathrm{m}^{2}\right)$ & $27.6 \pm 6.2$ & $26.4 \pm 6$ & $26.0 \pm 5.7$ & 0.068 & 0.144 & 0.043 \\
\hline Plasma glucose (mg/dL) & $161.1 \pm 59.4$ & $133.9 \pm 40.6$ & $131.3 \pm 44.7$ & 0.074 & 0.754 & 0.078 \\
\hline Plasma BNP (pg/mL) & $7.9 \pm 7.9$ & $17 \pm 16.9$ & $10.5 \pm 8.5$ & 0.012 & 0.272 & 0.109 \\
\hline Serum insulin & $9.4 \pm 5.2$ & - & $6.6 \pm 3.8$ & - & - & 0.075 \\
\hline Skeletal muscle mass $(\mathrm{kg})$ & $26.7 \pm 4.1$ & $25.8 \pm 4.3$ & $26.7 \pm 4.3$ & 0.293 & 0.080 & 0.893 \\
\hline Fat mass $(\mathrm{kg})$ & $25.7 \pm 10.3$ & $23 \pm 11.4$ & $22.1 \pm 11$ & 0.046 & 0.144 & 0.043 \\
\hline
\end{tabular}

Data are expressed as mean \pm SD. BNP: B-type natriuretic peptide; BMI: body mass index.

tes, who were administered metformin monotherapy. We excluded patients with heart failure and/or renal impairment. In addition, patients who took antihypertensive agents such as angiotensin converting enzyme inhibitors, angiotensin II receptor blockers, and $\beta$-blockers were excluded. Patients received 500 $-1,000 \mathrm{mg}$ per day of metformin as monotherapy for 6 months. We did not include any other antihyperglycemic agents during the study period. Plasma glucose, $\mathrm{HbAlc}$, BNP, serum insulin levels, and body composition were measured at baseline, and at 3 and 6 months after metformin administration.

\section{Treatment with SGLT2 inhibitors}

We studied seven outpatients with type 2 diabetes who were administered SGLT2 inhibitors in combination with other oral glucose-lowering drugs or insulin. We excluded patients with severe renal impairment (estimated glomerular filtration rate $(\mathrm{eGFR})<45 \mathrm{~mL} / \mathrm{min} / 1.73 \mathrm{~m}^{2}$ ) because the effect of SGLT2 inhibitors might decrease with increasing renal impairment [11]. We did not change insulin dose, other antihyperglycemic agents, antihypertensive agents, and cholesterol-lowering agents during the study period. Plasma glucose, HbAlc, BNP, and serum insulin levels were measured at baseline and approximately 3 months after starting SGLT-2 inhibitors. We also measured body composition and visceral fat area (VFA) in three of seven patients at baseline, and 3 months after the administration of SGLT-2 inhibitors.

The study protocol was approved by the Medical Ethics Committee of the National Center for Global Health and Medicine (Reference No. NCGM-G-001912), and the study was performed in accordance with the Declaration of Helsinki.

\section{Blood examination and body composition analysis}

We measured plasma glucose, HbA1c, BNP, and serum insulin levels. Plasma glucose level was measured using an enzymatic method (Wako Pure Chemical Industries, Osaka, Japan). Serum insulin and $\mathrm{HbA1c}$ levels were measured by automated en- zyme-linked immunosorbent assays (TOSOH, Tokyo, Japan). The eGFR was calculated using the revised equation adjusted for the Japanese population [12]. Plasma BNP levels were measured using a specific immunoradiometric assay for human BNP (ARCHITECT BNP-JP ${ }^{\circledR}$, Abbott Japan Co., Ltd, Tokyo, Japan). Body composition was analyzed using a bioelectrical impedance analysis device (InBody720, Biospace Co., Ltd, Tokyo, Japan). The VFA was measured using another bioelectrical impedance analysis device (InBodyS10, Biospace Co., Ltd, Tokyo, Japan). This method is based on the principle that lean body mass contains higher water and electrolyte content than fat tissue does, so these tissues can be distinguished by their electrical impedances. Segmental body composition was estimated using a patented eight-point tactile electrode system. The device uses six frequencies $(1,5,50,250,500$, and 1,000 kHz) and produces 30 impedance values for five body segments: the right and left upper extremities, trunk, and right and left lower extremities [13]. The VFA was also evaluated using this method. Previous validation studies using this device showed that both fat mass and lean body mass were highly correlated with those measured by dual-energy X-ray absorptiometry [14].

\section{Statistical analysis}

All statistical analyses were performed using SPSS version 22 (IBM Co., Ltd, Chicago, USA). All values were expressed as mean \pm standard deviation (SD). The Wilcoxon signed-rank test was applied to evaluate changes in plasma BNP levels, weight, body mass index (BMI), plasma glucose levels, plasma HbA1c levels, body fat mass, and skeletal muscle mass. A value of $\mathrm{P}<0.05$ was considered statistically significant.

\section{Results}

\section{Effects of metformin treatment}

Seven men and eight women with mean age of $54.9 \pm 14$ 
Table 2. Baseline Characteristics of Subjects Treated With SGLT2 Inhibitors

\begin{tabular}{|ll|}
\hline Baseline characteristics & \\
\hline $\mathrm{n}$ & 7 \\
Sex (men/women) & $3 / 4$ \\
Age (years) & $52.1 \pm 10.8$ \\
Duration of diabetes (years) & $5.6 \pm 5.5$ \\
Medication & \\
$\quad$ Metformin & 6 \\
$\quad$ Pioglitazone & 2 \\
$\quad$ DPP4 inhibitors & 3 \\
$\quad$ Sulfonylurea & 1 \\
$\quad$ Insulin & 1 \\
\hline
\end{tabular}

Data are expressed as number or mean \pm SD. SGLT2: sodium-glucose transporter 2; DPP4: dipeptidyl peptidase 4.

years were studied. Plasma BNP levels significantly increased 3 months after the initiation of metformin treatment. Weight, BMI, plasma glucose levels, and eGFR did not change significantly during the study period. However, HbA1c levels significantly decreased in the first 3 months of the study. Moreover, fat mass significantly decreased at both 3 and 6 months after metformin administration compared to the baseline value. In contrast, skeletal muscle mass did not change during the study period. The characteristics of the subjects and the results are summarized in Table 1.

\section{Effects of SGLT2 inhibitors}

Three men and four women with mean age of $52.1 \pm 10.8$ years and mean BMI of $32.3 \pm 6.7 \mathrm{~kg} / \mathrm{m}^{2}$ were studied. Five patients received $50 \mathrm{mg}$ of ipragliflozin, and two patients received 5 $\mathrm{mg}$ of dapagliflozin combined with $10 \mathrm{mg}$ of empagliflozin. The characteristics of subjects at baseline are listed in Table 2. Plasma BNP levels significantly increased 3 months after the addition of SGLT2 inhibitors to the treatment. The weight, BMI, eGFR, and plasma glucose and serum insulin levels did not change significantly within 3 months of starting treatment with SGLT2 inhibitors. However, HbA1c levels significantly decreased during the study period (Table 3 ). Although muscle mass did not change, fat mass and VFA decreased 3 months after the addition of SGLT2 inhibitors (ipragliflozin, dapagliflozin, and empagliflozin, respectively) to the treatment of three patients (Fig. 1).

\section{Discussion}

In the present study, we demonstrated that metformin monotherapy and treatment with SGLT2 inhibitors might increase plasma BNP levels within 3 months. To our knowledge, this is the first study to show the association of BNP levels with metformin monotherapy and treatment with SGLT2 inhibitors.
Table 3. Anthropometric and Biochemical Parameters at Baseline and 3 Months After SGLT2 Inhibitors Administration

\begin{tabular}{llll}
\hline & Baseline & After 3 months & P value \\
\hline $\mathrm{n}(\mathrm{men} /$ women) & $7(3 / 4)$ & - & - \\
Weight $(\mathrm{kg})$ & $85.4 \pm 20.1$ & $83.7 \pm 20.3$ & 0.063 \\
BMI $\left(\mathrm{kg} / \mathrm{m}^{2}\right)$ & $32.3 \pm 6.7$ & $31.6 \pm 6.6$ & 0.063 \\
Plasma glucose $(\mathrm{mg} / \mathrm{dL})$ & $151.0 \pm 64.5$ & $124.7 \pm 24.2$ & 0.108 \\
HbA1c $(\%)$ & $7.9 \pm 1.9$ & $7.2 \pm 1.1$ & 0.018 \\
Plasma BNP (pg/mL) & $8.8 \pm 7.2$ & $15.5 \pm 14.3$ & 0.018 \\
Serum insulin & $14.0 \pm 5.8$ & $11.5 \pm 3.3$ & 0.080 \\
\hline
\end{tabular}

Data are expressed as mean \pm SD. BNP: B-type natriuretic peptide; BMI: body mass index.

\section{BNP and metformin}

Plasma BNP levels were reported to be inversely related to BMI, waist circumference, and serum insulin levels in individuals without heart failure, which suggests that BNP is beneficially associated with insulin resistance [15]. However, we did not observe a significant change in serum insulin levels during the study period. Plasma BNP levels did not change in parallel with the change in serum insulin levels. In contrast, the decrease in fat mass was significant in the first 3 months of the study, after starting metformin treatment, although body weight and BMI did not change significantly. Rodriguez-Moctezuma et al showed that the administration of metformin decreased the fat mass and increased the lean mass [16]. Metformin may reduce fat mass by regulating $\mathrm{AMP}$-activated protein kinase (AMPK) [17]. Given that plasma BNP levels were negatively associated with visceral fat [18], a decrease in fat mass mediated by metformin might have increased plasma BNP levels in the first 3 months. Furthermore, BNP induces adipose tissue lipolysis in both healthy subjects and patients with heart failure [19]. Lower BNP levels have been reported to be due to increased clearance by the natriuretic peptide receptors in the adipose tissue [20]. Despite the non-significant decrease in fat mass between 3 and 6 months after starting metformin treatment, weight and fat mass significantly decreased across the study period, suggesting that increased AMPK activity by metformin could improve ectopic fat accumulation [21]. Therefore, short-term variation in plasma BNP levels may be caused by fat mass loss resulting from metformin treatment.

\section{BNP and SGLT2 inhibitors}

Loss of body weight following administration of SGLT2 inhibitors is because of reduced fat mass, and visceral and subcutaneous adipose tissue $[22,23]$. Yokono et al showed that treatment of high-fat diet-induced obese rats with ipragliflozin, an SGLT2 inhibitor, caused a decrease in body weight accompanied by reduced visceral and subcutaneous fat mass, but not lean mass [24]. They suggested that ipragliflozin enhanced lipolysis and fatty acid oxidation, as indicated by elevated plasma non-esterified fatty acid and 3-hydroxybutyrate 

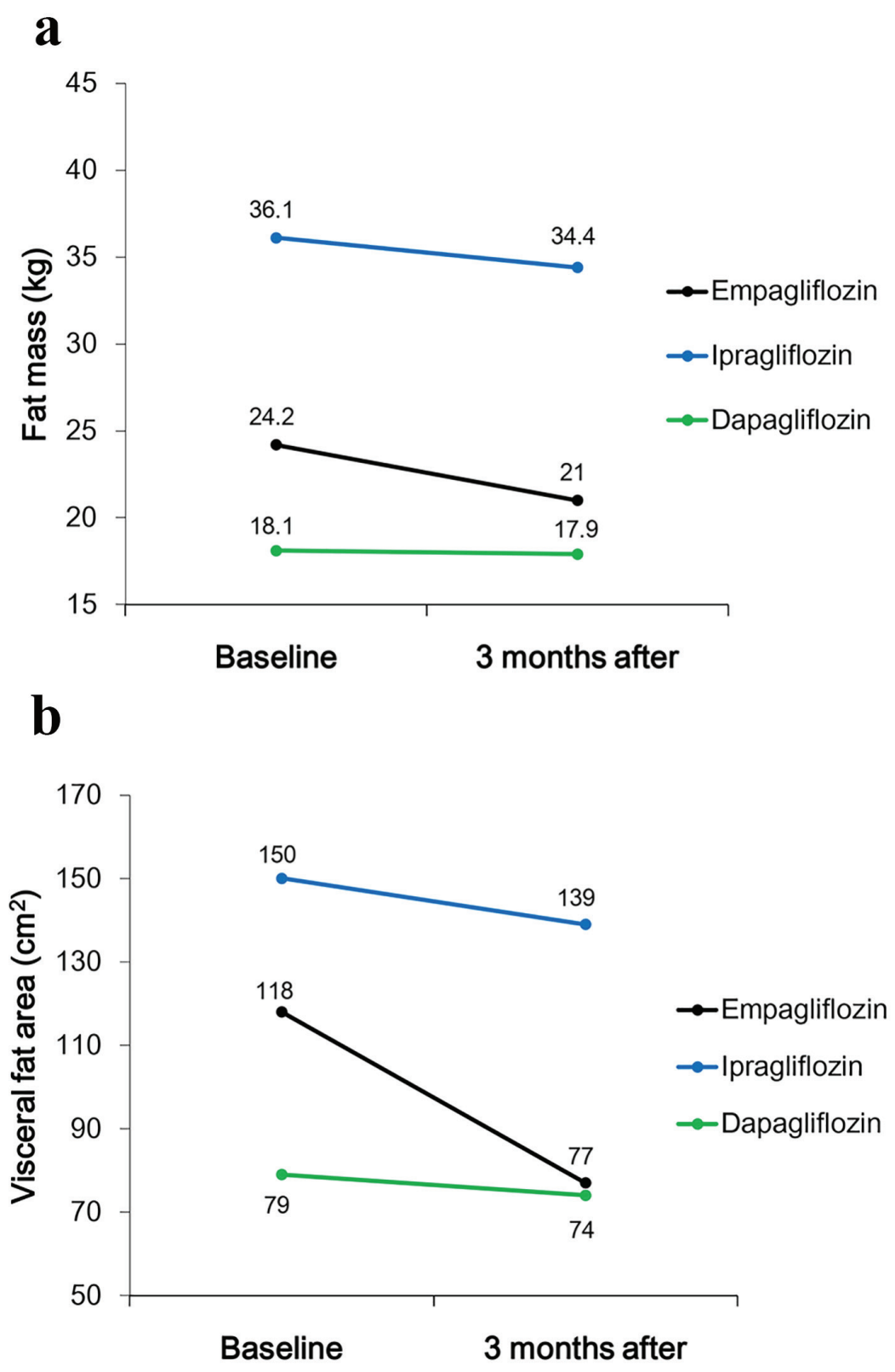

Figure 1. Changes in (a) fat mass and (b) visceral fat area (VFA) between baseline and 3 months after starting treatment with SGLT2 inhibitors.

levels, and urinary excretion of 3-hydroxybutyrate [24]. In the present study, three patients showed decreased fat mass and VFA accompanied by increased plasma BNP levels after treatment with SGLT2 inhibitors, suggesting that SGLT2 inhibitors increase plasma BNP levels, and induce adipose tissue lipolysis mediated by BNP [19]. Furthermore, natriuretic peptides including BNP have cardioprotective effects. They attenuate the cardiac hypertrophic action of angiotensin II, and suppress cardiac sympathetic nerve activity in humans [25]. One reason why cardiovascular events decreased within 3 months in patients treated with empagliflozin in the EMPA-REG OUTCOME trial could be explained by the cardioprotective effect of elevated BNP caused by empagliflozin treatment.
However, current literature on this issue is very limited, and the associations between BNP level and cardioprotective effects of metformin and SGLT2 inhibitors are still unknown. Further investigations are required.

\section{Limitations}

This study is a small single-center observational study and does not have a control group. Therefore, the study data are insufficient to derive a definitive conclusion concerning the effects of metformin and SGLT2 inhibitors on plasma BNP levels. Changes in dietary intake and physical activity might 
have affected the study results. Lack of data concerning serum insulin levels in patients treated with metformin, and body composition in patients treated with SGLT2 inhibitors, is another study limitation. We could not measure body composition in all study subjects. However, body composition analysis led to the hypothesis that adipose tissue lipolysis mediated by BNP level has a role in loss of fat mass and the administration of metformin and SGLT2 inhibitors provides cardioprotection.

\section{Conclusion}

Plasma BNP levels increased as a result of fat mass loss induced by the administration of metformin and SGLT2 inhibitors within 3 months of starting treatment. Our results suggest that metformin and SGLT2 inhibitors could reduce the risk of CVD through the cardioprotective effects of BNP in patients with type 2 diabetes.

\section{Conflict of Interest}

The authors declare no conflicts of interest.

\section{Funding}

We have not received any funding or grants for this study.

\section{References}

1. Mozaffarian D, Benjamin EJ, Go AS, Arnett DK, Blaha MJ, Cushman M, de Ferranti S, et al. Heart disease and stroke statistics - 2015 update: a report from the American Heart Association. Circulation. 2015;131(4):e29-322.

2. Holman RR, Sourij H, Califf RM. Cardiovascular outcome trials of glucose-lowering drugs or strategies in type 2 diabetes. Lancet. 2014;383(9933):2008-2017.

3. Effect of intensive blood-glucose control with metformin on complications in overweight patients with type 2 diabetes (UKPDS 34). UK Prospective Diabetes Study (UKPDS) Group. Lancet. 1998;352(9131):854-865.

4. Calvert JW, Gundewar S, Jha S, Greer JJ, Bestermann WH, Tian R, Lefer DJ. Acute metformin therapy confers cardioprotection against myocardial infarction via AMPK-eNOS-mediated signaling. Diabetes. 2008;57(3):696705.

5. Cadeddu C, Nocco S, Deidda M, Cadeddu F, Bina A, Demuru P, Cossu E, et al. Cardiopulmonary and endothelial effects of metformin treatment in an insulin resistant population. Int J Cardiol. 2012;158(2):302-304.

6. Abdul-Ghani MA, Norton L, DeFronzo RA. Efficacy and safety of SGLT2 inhibitors in the treatment of type 2 diabetes mellitus. Curr Diab Rep. 2012;12(3):230-238.

7. Devineni D, Morrow L, Hompesch M, Skee D, Vandebosch A, Murphy J, Ways K, et al. Canagliflozin improves glycaemic control over 28 days in subjects with type 2 diabetes not optimally controlled on insulin. Diabetes Obes Metab. 2012;14(6):539-545.

8. Zinman B, Wanner C, Lachin JM, Fitchett D, Bluhmki E, Hantel S, Mattheus M, et al. Empagliflozin, Cardiovascular Outcomes, and Mortality in Type 2 Diabetes. N Engl J Med. 2015;373(22):2117-2128.

9. Albertini JP, Cohen R, Valensi P, Sachs RN, Charniot JC. B-type natriuretic peptide, a marker of asymptomatic left ventricular dysfunction in type 2 diabetic patients. Diabetes Metab. 2008;34(4 Pt 1):355-362.

10. Wang TJ, Larson MG, Levy D, Benjamin EJ, Leip EP, Omland T, Wolf PA, et al. Plasma natriuretic peptide levels and the risk of cardiovascular events and death. N Engl J Med. 2004;350(7):655-663.

11. Sarashina A, Ueki K, Sasaki T, Tanaka Y, Koiwai K, Sakamoto W, Woerle HJ, et al. Effect of renal impairment on the pharmacokinetics, pharmacodynamics, and safety of empagliflozin, a sodium glucose cotransporter 2 inhibitor, in Japanese patients with type 2 diabetes mellitus. Clin Ther. 2014;36(11):1606-1615.

12. Matsuo S, Imai E, Horio M, Yasuda Y, Tomita K, Nitta $\mathrm{K}$, Yamagata $\mathrm{K}$, et al. Revised equations for estimated GFR from serum creatinine in Japan. Am J Kidney Dis. 2009;53(6):982-992.

13. Anderson LJ, Erceg DN, Schroeder ET. Utility of multifrequency bioelectrical impedance compared with dualenergy x-ray absorptiometry for assessment of total and regional body composition varies between men and women. Nutr Res. 2012;32(7):479-485.

14. Faria SL, Faria OP, Cardeal MD, Ito MK. Validation study of multi-frequency bioelectrical impedance with dual-energy X-ray absorptiometry among obese patients. Obes Surg. 2014;24(9):1476-1480.

15. Wang TJ, Larson MG, Levy D, Benjamin EJ, Leip EP, Wilson PW, Vasan RS. Impact of obesity on plasma natriuretic peptide levels. Circulation. 2004;109(5):594-600.

16. Rodriguez-Moctezuma JR, Robles-Lopez G, Lopez-Carmona JM, Gutierrez-Rosas MJ. Effects of metformin on the body composition in subjects with risk factors for type 2 diabetes. Diabetes Obes Metab. 2005;7(2):189-192.

17. Rojas J, Arraiz N, Aguirre M, Velasco M, Bermudez V. AMPK as Target for Intervention in Childhood and Adolescent Obesity. J Obes. 2011;2011:252817.

18. Yamashita T, Kohara K, Tabara Y, Ochi M, Nagai T, Okada Y, Igase M, et al. Muscle mass, visceral fat, and plasma levels of B-type natriuretic peptide in healthy individuals (from the J-SHIPP Study). Am J Cardiol. 2014;114(4):635-640.

19. Polak J, Kotrc M, Wedellova Z, Jabor A, Malek I, Kautzner J, Kazdova L, et al. Lipolytic effects of B-type natriuretic peptide 1-32 in adipose tissue of heart failure patients compared with healthy controls. J Am Coll Cardiol. 2011;58(11):1119-1125.

20. Das SR, Drazner MH, Dries DL, Vega GL, Stanek HG, Abdullah SM, Canham RM, et al. Impact of body mass and body composition on circulating levels of natriuretic peptides: results from the Dallas Heart Study. Circulation. 2005;112(14):2163-2168.

21. Malin SK, Kashyap SR. Effects of metformin on weight 
loss: potential mechanisms. Curr Opin Endocrinol Diabetes Obes. 2014;21(5):323-329.

22. Bolinder J, Ljunggren O, Kullberg J, Johansson L, Wilding J, Langkilde AM, Sugg J, et al. Effects of dapagliflozin on body weight, total fat mass, and regional adipose tissue distribution in patients with type 2 diabetes mellitus with inadequate glycemic control on metformin. J Clin Endocrinol Metab. 2012;97(3):1020-1031.

23. Bolinder J, Ljunggren O, Johansson L, Wilding J, Langkilde AM, Sjostrom CD, Sugg J, et al. Dapagliflozin maintains glycaemic control while reducing weight and body fat mass over 2 years in patients with type 2 diabetes mellitus inadequately controlled on metformin. Diabetes Obes Metab. 2014;16(2):159-169.

24. Yokono M, Takasu T, Hayashizaki Y, Mitsuoka K, Kihara R, Muramatsu Y, Miyoshi S, et al. SGLT2 selective inhibitor ipragliflozin reduces body fat mass by increasing fatty acid oxidation in high-fat diet-induced obese rats. Eur J Pharmacol. 2014;727:66-74.

25. Woods RL. Cardioprotective functions of atrial natriuretic peptide and B-type natriuretic peptide: a brief review. Clin Exp Pharmacol Physiol. 2004;31(11):791-794. 